\section{One cloud and its silver lining}

\section{J. D. Lowenthal}

DisTANT, luminous quasistellar objects shine through multitudes of otherwise invisible intergalactic clouds of hydrogen that carve a forest of Lyman- $\alpha$ absorption lines out of the background sources' spectra. Unfortunately, because the background sources are typically pointlike, we usually get no information on the size or form of the foreground clouds: are they flat or round, the size of galaxies or of the Solar System, neutral or ionized? On page 224 of this issue ${ }^{1}$, however, Hippelein and Meisenheimer present the results of a clever observation in which they imaged in absorption a putative Lyman- $\alpha$ forest cloud in front of a distant radio galaxy; because radio galaxies are spatially extended, this experiment yields a direct measurement of the cloud's physical shape and size, which corresponds roughly to the dimensions of large local galaxies.

The issue bears strongly on various pictures of galaxy formation and cosmology: because of the finite speed of light, we see the Lyman- $\alpha$ clouds as they were when the Universe was very young, so that knowing their basic physical parameters will clarify their relation to normal galaxies. It will also provide insight into such important issues as the strength and source of the intergalactic ionizing radiation field.

The size of Lyman- $\alpha$ clouds is crucial for understanding their ionization states, abundance of elements heavier than helium (metals) and gas densities, but cannot be obtained by analysis of the spectra of the quasistellar objects (QSOs). The multiple images of rare, gravitationally lensed QSOs offer one way forward. Each image corresponds to a distinct path followed by the QSO's radiation. A small cloud may appear on a single image, but a large one may intercept several paths so that its size may be estimated. The most carefully studied $^{2}$ gravitationally lensed QSO case to date, UM673 A and B, yields typical cloud sizes in the range 10-200 kiloparsecs, depending on the interpretation of a few particular absorption lines and on cosmological parameters. This agrees with earlier similar studies ${ }^{3}$ and is reminiscent of normal galactic dimensions today. But a contrasting suggestion, based on a possible correlation between Doppler spectral line widths and column densities, and on the observed narrowness of some absorption lines ${ }^{4}$, is that the clouds are cold and minuscule (less than a parsec) or sheet-like ${ }^{5,6}$.

Hippelein and Meisenheimer's observations make use of two important characteristics of the Lyman- $\alpha$ emission line in the spectrum of the luminous, high-redshift ( $z=3.4$, corresponding to a lookback time of at least 80 per cent of the age of the Universe) radio galaxy $4 \mathrm{C} 41.17$ (ref. 7). First, the radiation comes from a spatially extended region, subtending some $10 \times 13$ arcseconds on the sky, or about 100 kiloparsecs at the radio galaxy's distance from us; and second, the emission line is spectrally very broad. Together, these facts cause the extended emission line region to appear as a light screen, a backdrop resembling a continuum source, against which any intervening neutral hydrogen cloud within a reasonable distance of the radio galaxy would appear as a dark Lyman- $\alpha$ absorption feature; if the intervening cloud were spatially extended, so would the absorption feature be. This is exactly what Hippelein and Meisenheimer report, in agreement with a previous, detailed study of the $4 \mathrm{C} 41.17$ system ${ }^{7}$.

Using a Fabry-Perot interferometer (a tuneable narrow-band filter) and an imaging charge-coupled device (CCD), Hippelein and Meisenheimer took images of the Lyman- $\alpha$ emission line, stepping the Fabry-Perot through consecutive wavelengths, mostly blue-shifted from the emission line (to see intervening absorption lines). At the wavelength corresponding to Lyman- $\alpha$ at a blueshift of about $360 \mathrm{~km} \mathrm{~s}^{-1}$ with respect to the emission-line maximum, they detect an oblong dark patch visible against the bright background. They propose that it is due to an intervening hydrogen cloud of dimensions $10 \times 14$ kiloparsecs, which could be 5 megaparsecs from the background source.

Assuming some nominal Lyman- $\alpha$ cloud parameters, Hippelein and Meisenheimer derive a column density of neutral hydrogen around $10^{15} \mathrm{~cm}^{-2}$, yielding for their measured cloud size a total mass of hydrogen of $3 \times 10^{7}$ solar masses. This would imply that the Lyman- $\alpha$ forest clouds at high redshift are the same size as modern-day galaxies, but much less dense, and are perhaps held together by their own gravity ${ }^{8}$. They could be elongated or possibly sheet-like, and have $10^{4}-10^{5}$ times as much ionized as neutral hydrogen. This picture is in good agreement with the implications of the earlier gravitationally lensed QSO studies ${ }^{4}$. The presence of any absorption lines due to metals would show that the cloud is not pristine and has been contaminated by a generation of star formation.

However, several caveats remain. In particular, the interpretation of the dark patch is far from unambiguous. As Hippelein and Meisenheimer point out, it could be due to complexities in the background emission (indeed, the Lyman- $\alpha$ emission cloud surrounding the radio galaxy is already known to be quite complex kinematically ${ }^{7}$ ). And as the authors recognize, even if the cloud is real, it might be strongly influenced by radiation from the powerful radio galaxy. Or it might even be directly associated with the radio galaxy itself and so not typical of Lyman- $\alpha$ forest clouds at all. This last possibility is supported by previous long-slit spectroscopic images ${ }^{7}$ of the Lyman- $\alpha$ line, which give higher resolution than do Hippelein and Meisenheimer's narrow-band images. As one would expect from the images, a dark absorption (or at least a lack-ofemission) feature is visible a few ångströms blueward of the emission line peak. The absorption line appears to have a velocity gradient of $120 \mathrm{~km} \mathrm{~s}^{-1}$, typical of galaxy rotation effects but larger than typical Lyman- $\alpha$ cloud velocity widths. Chambers et al. ${ }^{7}$ refer to this feature as a disk, and it is presumably the same object or component that Hippelein and Meisenheimer imaged. The inferred mass, if the velocity shear represents a circular orbit driven by gravity, is around $3 \times 10^{11}$ solar masses (or a bit less, depending on the inclination of the disk to the observer); this is clearly different from the mass given by Hippelein and Meisenheimer.

There are several other high-redshift, luminous radio galaxies with extended regions of Lyman- $\alpha$ emission: if these typically show dark patches similar to that in the spectrum of $4 \mathrm{C} 41.17$, the natural conclusion would be that the absorbing material is linked directly to the parent galaxy, either in the form of a surrounding ring or shell or as a companion galaxy or cloud. If so we would stand, at least, to learn much about distant radio galaxies, which may in turn hold clues to normal galaxy formation. But if the absorbing cloud turns out to be representative of the Lyman- $\alpha$ forest, the implications for our understanding of the clouds' physical parameters will be far-reaching.

J. D. Lowenthal is at the Space Telescope Science Institute, 3700 San Martin Drive, Baltimore, Maryland 21218, USA.

\footnotetext{
1. Hippelein, H. \& Meisenheimer, K. Nature 362, 224-226 (1993)

2. Smette, A. et al. Astrophys. J. 389, 39-62 (1992)

3. Foltz, C. B. et al. Astrophys. J. 281, L1-L4 (1984)

4. Pettini, M., Hunstead, R. W., Smith, L. J. \& Mar, D. P. Mon. Not. R. astr. Soc. 246, 545-564 (1990).

5. Carswell, R. F., Lanzetta, K. M., Parnell, H. C. \& Webb, J. K. Astrophys. J. 371, 36-48 (1991).

6. Hogan, C. J. Astrophys. J. 316, L59-L61 (1987).

7. Chambers, K. C., Miley, G. K. \& van Breugel, W. J. M. Astrophys. J. 363, 21-39 (1990).

8. Charlton, J. C., Salpeter, E. E. \& Hogan, C. J. Astrophys J. 402, 493-513 (1993)
} 